International Journal of Advanced Research in Engineering and Technology (IJARET)

Volume 11, Issue 7, July 2020, pp. 363-371, Article ID: IJARET 1107036

Available online athttp://iaeme.com/Home/issue/IJARET?Volume $=\overline{1} 1 \& \overline{\mathrm{I}} \mathrm{ssue}=7$

ISSN Print: 0976-6480 and ISSN Online: 0976-6499

DOI: 10.34218/IJARET.11.7.2020.036

(C) IAEME Publication

Scopus Indexed

\title{
AN EFFICIENT SELFISHNESS CONTROL MECHANISM FOR MOBILE AD HOC NETWORKS
}

\author{
D. Rajalakshmi \\ Research Scholar, Department of Computer Science and Engineering \\ Vel Tech Rangarajan Dr. Sagunthala R\&D Institute of Science and Technology, \\ Chennai, Tamilnadu, India. \\ Assistant Professor, Department of Computer Science and Engineering \\ Sri Sairam Institute of Technology, Chennai, Tamilnadu, India.

\section{Dr. K. Meena} \\ Professor, Department of Computer Science and Engineering \\ Vel Tech Rangarajan Dr.Sagunthala R\&D Institute of Science and Technology, \\ Chennai, Tamilnadu, India.
}

\begin{abstract}
A MANETs (mobile ad hoc network) is a self-organized wireless network. This network is more vulnerable to security failure because due to dynamic topology, infrastructure less environment and energy consumption. Based on this security issue routing in MANET is very difficult in real time perspectives. In these kinds of networks, the mobility and resource constraints could leads to divide the networks and minimizing the performance of entire network. In real time it's not possible because some selfish nodes it interacts with other nodes partially or it may not share the data entirely. These kind of malicious or selfish nodes it degrades the network performance. In this paper, proposed and implemented the effect of malicious activities in a MANETs, using Self Centered Friendship tree Routing. It's a novel replica model, motivated by the Social relationship. Using this technique it detects the malicious nodes, and prevent the hacking issues in routing protocol in future routes.
\end{abstract}

Key words: Intrusion detection, Misbehaving Nodes, Routing, Replica Allocation, Security and Selfish Nodes.

Cite this Article: D. Rajalakshmi and Dr. K. Meena, An Efficient Selfishness Control Mechanism for Mobile Ad Hoc Networks, International Journal of Advanced Research in Engineering and Technology, 11(7), 2020, pp. 363-371.

http://iaeme.com/Home/issue/IJARET?Volume=11\&Issue=7 


\section{INTRODUCTION}

A Mobile ad hoc network (MANET) is a collection of devices; it creates the temporary connection without any infrastructure. It communicates the other nodes via multi-hop. No master slave relationship applicable in MANETs [1].

Due to dynamic topology, the devices are simply entered the network and perform the communication then its moves the network in a very fashionable manner. The network size and topology is unpredictable because of this infrastructure less environment [2]. The network performance is highly solicited on the entire nodes. It is mainly used in following applications: Military, Rescue operation, Business work, class rooms, conference and Personal Area Network (PAN).

In MANET, the devices are simply move from one place to another place arbitrarily without any difficulties. Individual node may act as a router, it by pass the network traffic to other nodes in the network. MANETs can easily form and deform the networks without the need of any centralized arbiter.

MANETs are classified into four types, namely:

1. VANET - Vehicular Ad hoc Network

2. IMANET - Internet Based Mobile Ad hoc Network

3. INVANET - Intelligent Vehicular Ad hoc Network

4. FANET - Flying Ad hoc Network

In MANETs the routing protocols are used, to deliver the packet to intended recipient. It consists of set of rules and regulations that governs communication from source to destination. There are three types of routing Protocol [3], namely:

1. Proactive Routing Protocol

2. Reactive Routing Protocol

3. Hybrid Routing Protocol

\subsection{Proactive Routing Protocol}

Each and every node should maintain a routing table periodically. Based on the network traffic the packets are delivered to the destination through some other neighbors. It's not a best approach for larger networks, because for all nodes maintaining a routing table is not an easy task. Examples: Destination Sequenced Distance Vector Routing (DSDV), Wireless Routing Protocol (WRP), Optimized Link State Routing Protocol (OLSR) etc.

\subsection{Reactive Routing Protocol}

Routing table is not updated periodically, that is whenever the network configuration has changed then only routing information is updated, and finds the optimal path to deliver the packets into corresponding destination. Examples: Ad-hoc On-demand Distance Vector (AODV), Dynamic Source Routing (DSR), Low Based Multipath Routing (LMR) etc.

\subsection{Hybrid Routing Protocol}

It combines best approaches of proactive and reactive routing protocols. Examples: Zone Routing Protocol (ZRP), Border Gateway Protocol (BGP) etc.

\section{BACKGROUND}

Due to the emerging era, MANET has populated in recent years. Without physical infrastructure, it performs the communication in very effective manner in the cases of dynamic technology configuration, energy consumption etc. The main characteristics of 
MANETs are fast installation, low bandwidth communication and narrow processing competency [4]. Based on the characteristics, it provides the routing information to the standard routing protocols. There are no rules and regulations for travelling the packets when inside the network region. So individual node they can choose the path accordingly to do the communication with one another [5]. Traditional Routing protocols are used in wireless communications and it gives the routing information to specified node. Some existing traditional routing protocols are AODV, OLSR, and DSR etc. Furthermore, each node has autonomously to detect and react on network topology changes [5]. In research point of view MANETs have several challenges in security perspectives. It has a limited battery power and limited communication area [6].

In large-scale network, the numbers of communication devices are low and the distance between one to another device becomes too high means the network falls into unreliable one and also some malfunctioning or misbehaviors are performed inside the network [7]. This is unfavorable, because the communication demands are very high in the recent technologies.

\section{RELATED WORK}

In Mobile Ad hoc region, reactive routing protocol is more robust. Individual selfishness communication node achieves low cost-efficiency and truthfulness. Ad hoc-VCG works well for ad hoc networks, where communication between two nodes is generally long and the routing path does not change intensely during a communication [8]. If these conditions are not met, overhead of the route discovery phase stalls the network performance.

In this paper, a game-theoretic approach is used to produce better results with the existing system approaches. It analyzes the problem through theoretical perspectives. In selfish caching model, servers have two possible actions for each object. If the replica of a requested object is located at nearby node, the server accesses the remote replica. If the replica of a requested object is located at remote node, server caches the data by the object itself. Decisions about the caching and replicas are performed at locally, with their local costs. If one or more servers to do the same operation means the object is replicated in all servers. All servers specify the game theoretic approach and calculate the relevant cost for all servers [9].

In existing method, the selfish nodes are handled by three categories [10-11]. The Reputation-based Technique monitors the user behaviors and gets the routing information for delivering the packets from sender to receiver [12-14]. The Credit-payment technique gives credit information to others through this kind of transformation data is shared to others. The Game theory-based technique identifies the equilibrium point to improve the system performance. Above mentioned three categories concentrated on forwarding of packets. But in proposed system, give attention to forwarding of packets, Packet delivery ratio, routing overhead, and identifying the selfishness node behavior and activities.

\section{Disadvantages of Existing System}

- It only focused on the packet forwarding.

- In DCG, network traffic is very worst.

\section{PROBLEM DEFINITION}

It illustrates the issues of selfishness perspective, replica provision in MANET. It can easily identified by peer-to-peer application. The System Architecture is represented in figure 1.

The SCF-tree consists of three parts: 1) Detecting the selfish nodes, 2) Build the SCF-tree, and 3) Replica Allocation. The SCF-tree model enhances the human relationship management in real world environments. 
The proposed technique is novel replica allocation for detecting the selfish node. In this method self-centered friendship tree (SCF-tree) concept is implemented. Main advantage of using this model is to achieve good efficiency with more scalability and low cost communication.

\section{Advantages of SCF Tree}

- It reduces the operational cost.

- Easy Access to the end users.

- Identify the selfish nodes in very effective manner.

In existing method they are focused on the issues of network and parameters [15-18]. That is, the selfish node does not send the information to other nodes to their own battery consumption. It provides more services to military and high-end security applications.

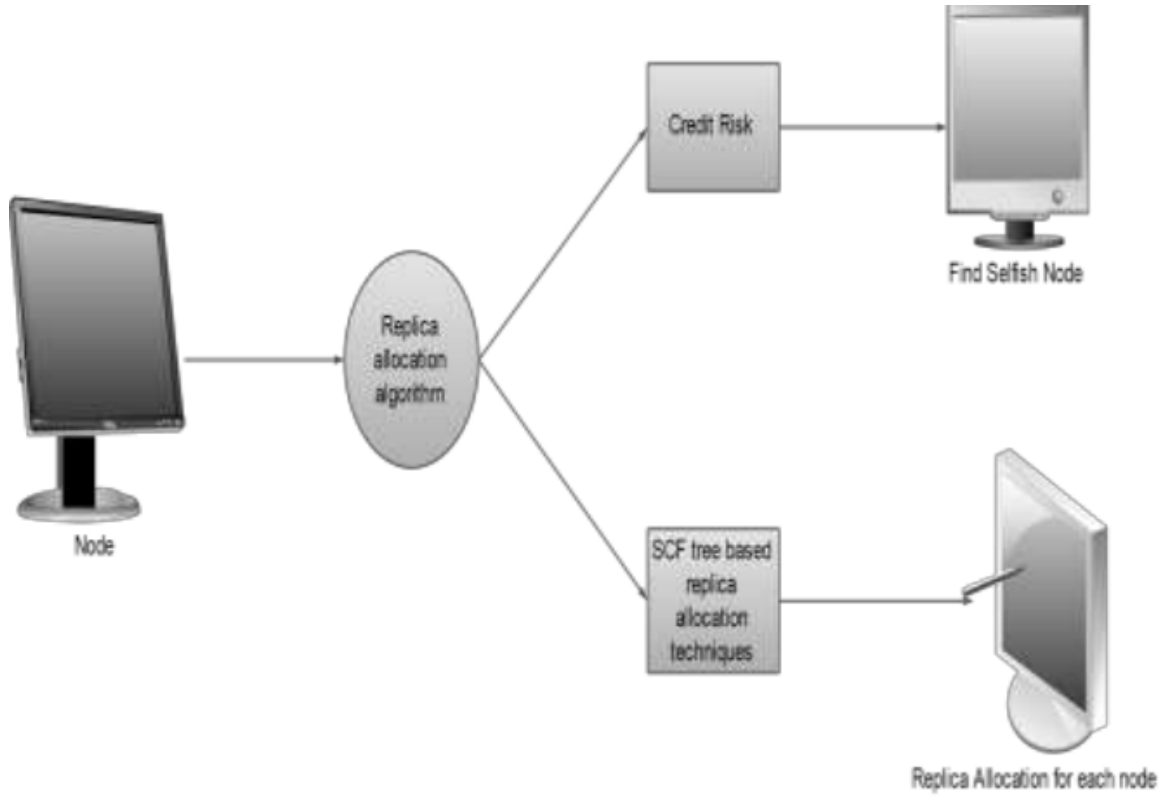

Figure 1 System Architecture

Using this Self-Centered- Friendship Tree, easily detect the full and partial selfish nodes effectively, allocating the replica effectively and Honest Grouping. The main goal of this HG (Honest Grouping) scheme is, it replicates the most needed data's only because it increase the availability and reduce the latency of the specified groups. In HG scheme, the node may want get the resource of others the node must be share its own resource (Give and Take policy). In this network arrangement the extreme support of collaboration is solicited due to no redundancy.

\section{PROPOSED SYSTEM}

\subsection{Self Centered Friendship Tree}

Self-Centered Friendship tree is a kind of novel replica method. It is motivated by the social relationship. In this method the neighbor's feedbacks are not considered to build the tree, it is a decision makes by its own experiments. Before constructing the tree, it requires to eliminate all selfishness nodes in the link. The node selected for replication is based on the route. The child nodes also check recursively. A node which has multiple nodes to the root node is selected for replication. SCF tree maintained the routing techniques. It is depicted in figure 2. 


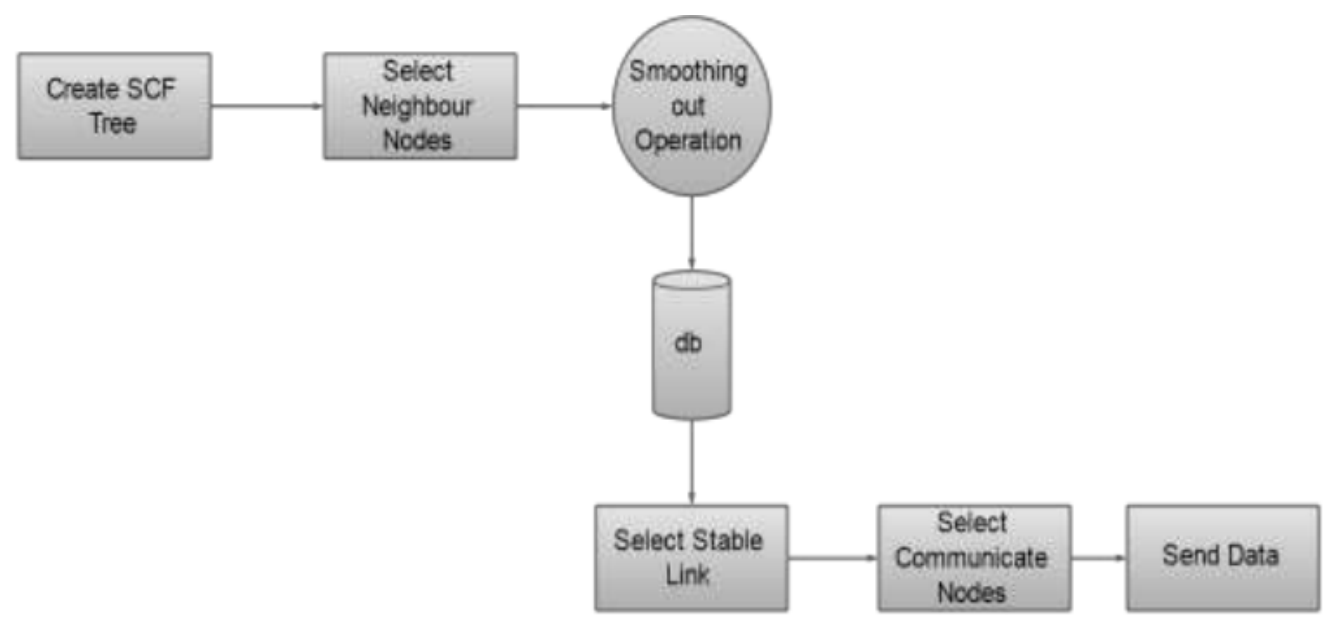

Figure 2 Self Centered Friendship Tree

\subsection{Smoothing Out Operation}

This concept is comes under the mathematical approach of graph based theory. It eliminates the mischievous node behavior and activities inside the network. It identifies the strength of the channel then only communication is performed between sender and receiver, otherwise it may not share the communication to other nodes. Whenever there's a change in network configuration every time it checks the channel strength then only communication is initiated.

\subsection{Replica Allocation}

Using this Self Centered Friendship tree, a node allocates replica for all non-selfish nodes, because it does not have enough space to store the data in internal memory. So, the individual node it store the data to the SCF tree in the basis of priority techniques. After selecting the replica node, first it checks the own memory space for placing replica file, if it not enough space another replica node will selected based on the next high frequency. Replica allocation technique is used to reduce the query delay and increases the data accessibility.

\subsection{Detecting Selfish Nodes}

Individual node can measure the selfishness node by the credit risk score and it finds the malicious node in that specified network zone.

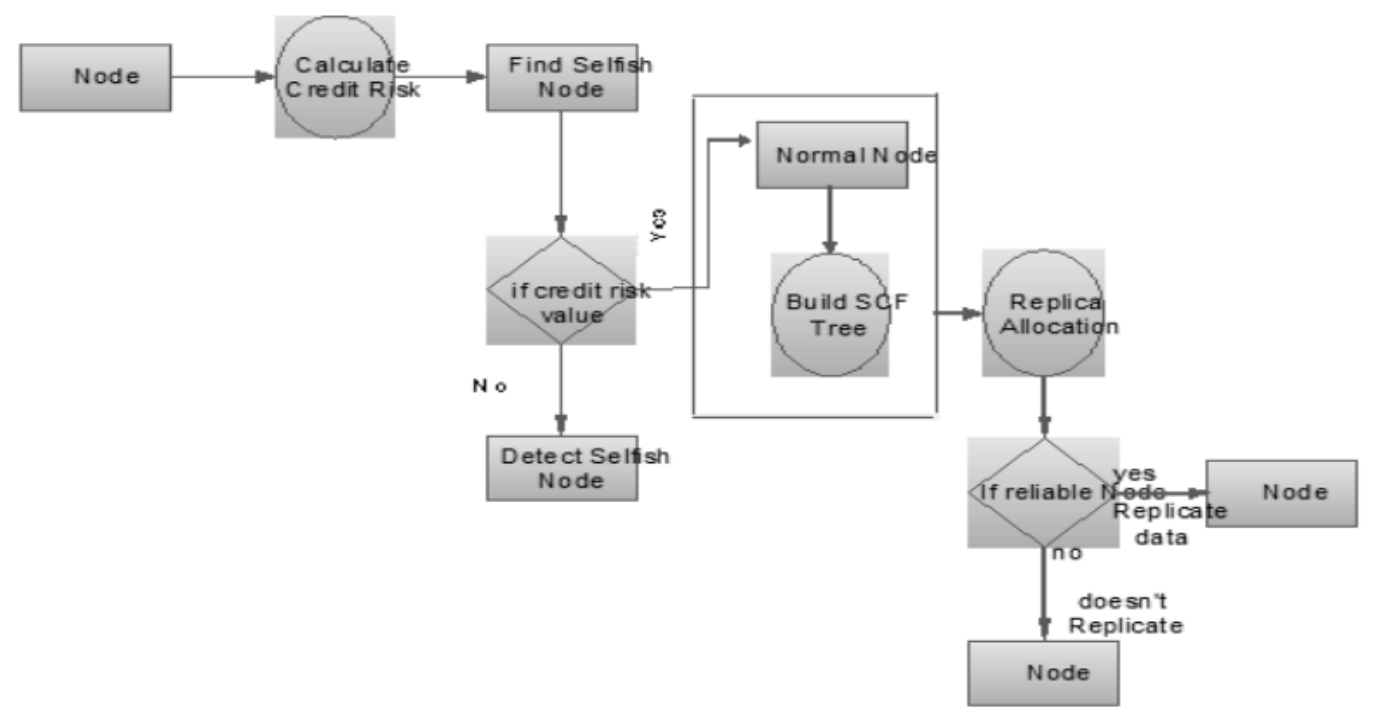

Figure 3 System Implementation for Detecting Selfish Nodes 
Credit risk is calculated by the regular range of credit risk values and predictable values. These selfish nodes are categorized into two types: 1.Unambiguous Node 2. Unambiguous Query processing. The Unambiguous Node categories node which is not shares the memory space or data items for others. The degree of the node is calculated by using shared data item. In the Unambiguous Query Processing categories node which is not a selfish node, it cannot give the file because of unexpected link failures. These may intimate the communicate parties by using selfishness alarm. It is represented in figure 3 .

\section{RESULT AND DISCUSSION}

In this section compare the results of existing methods and protocols like AODV, DSR with the proposed system, which is self-centered friendship tree with the following parameters: Packet delivery ratio, Routing overhead, Source to Destination delay and lifetime of the network. Comparatively with existing methods, the SCF tree is robust and the performance also improved in real time applications. Table.1 shows the Comparisons of AODV, DSR and SCF Tree in different parameters.

Table 1 Comparisons of AODV, DSR and SCF Tree

\begin{tabular}{|l|c|c|c|}
\hline \multicolumn{1}{|c|}{ Parameters } & AODV & DSR & SCF Tree \\
\hline Packet Delivery Ratio & Average & Average & High \\
\hline Routing Overhead & Low & Low & Average \\
\hline $\begin{array}{l}\text { Source to Destination } \\
\text { Delay }\end{array}$ & Moderate & Moderate & Low \\
\hline Lifetime of the Network & Moderate & Moderate & Good \\
\hline
\end{tabular}

\subsection{Packet Delivery Ratio}

The packet delivery ratio is the number of packets transmitted from source to destination, then how many packets are received in the destination without any loss, this ratio is calculated by packet delivery ratio and in SCF it becomes high, when compared to AODV and DSR. It's shown in Figure 4.

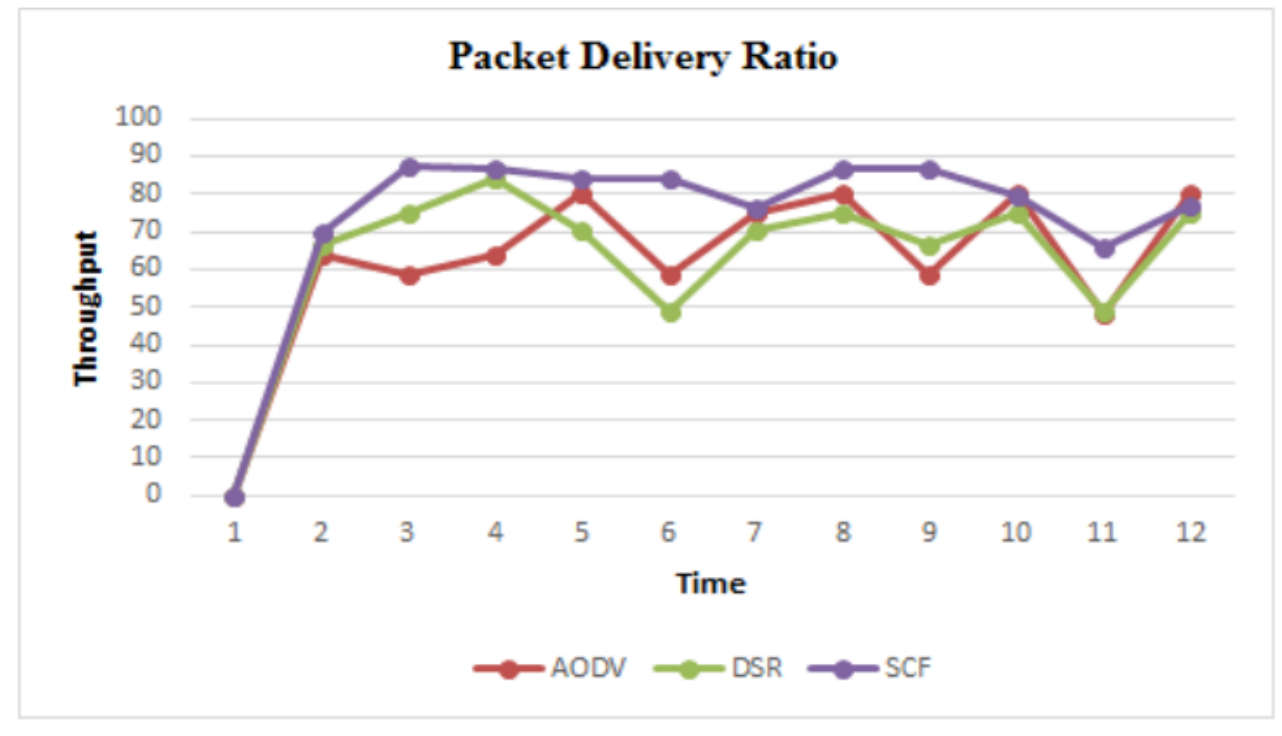

Figure 4 Packet Delivery Ratio 


\subsection{Routing Overhead}

In MANETs the routing path becomes divided and finding the optimal path to deliver the packets from source to destination. Due to dynamic topology changes, generally the routing overhead is high, but while using SCF, the routing overhead is reduced and the throughput is increased. It's shown in Figure 5.

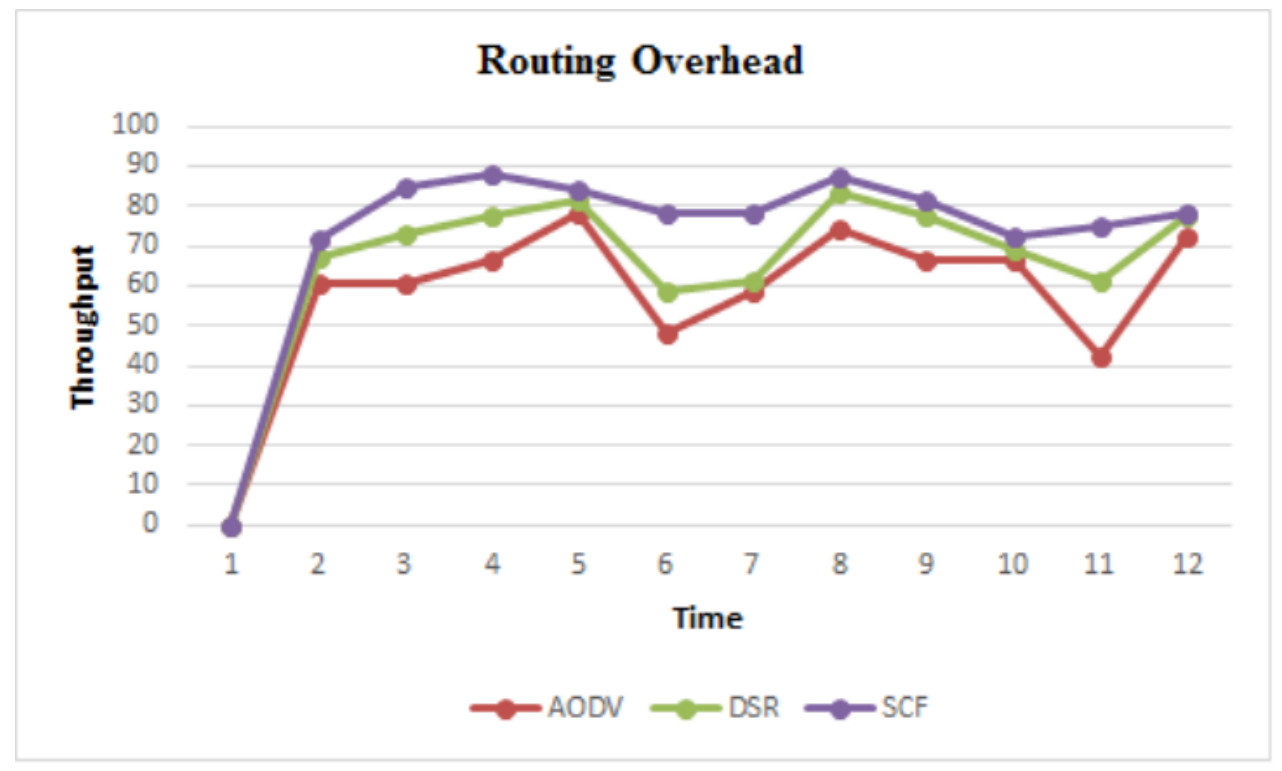

Figure 5 Routing Overhead

\subsection{Source to Destination Delay}

In MANET's time taken to forward and receive the packets from intended sender to intended receiver, the total time consumption is source to destination delay or End to End delay. It's shown in Figure 6.

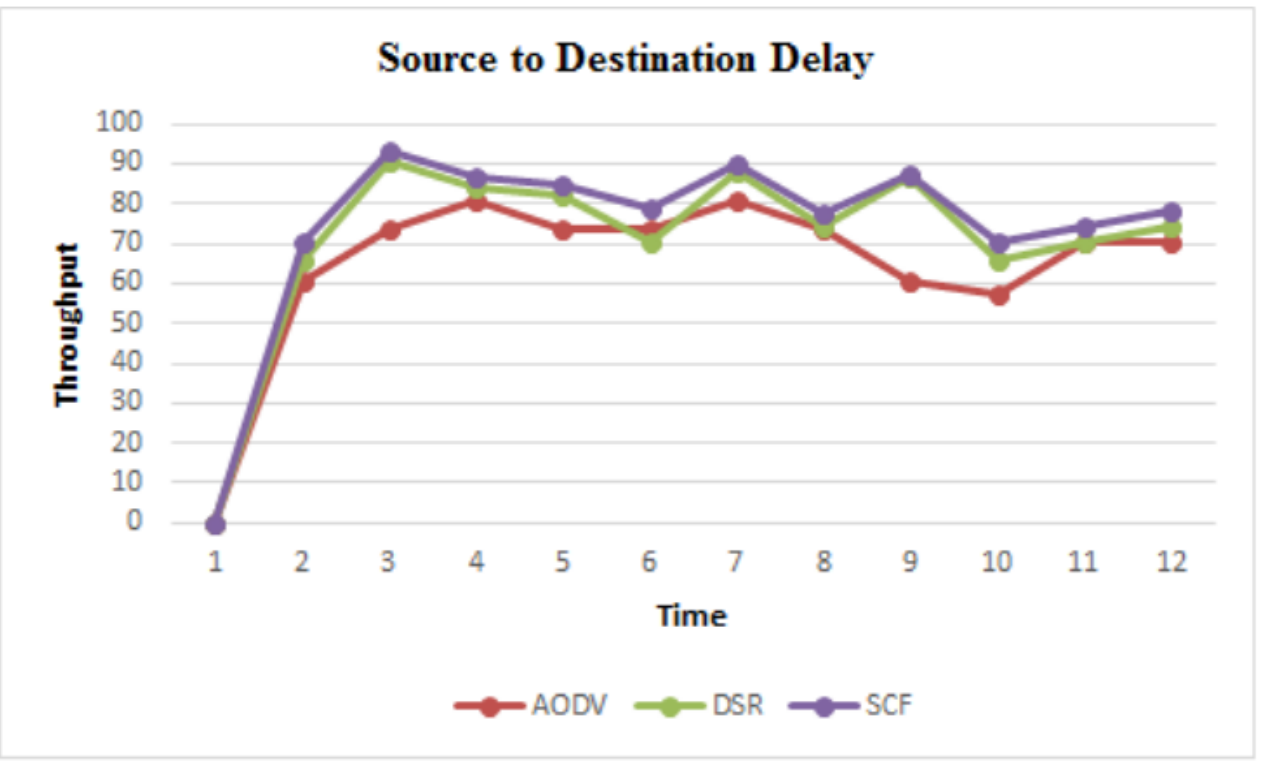

Figure 6 Source to Destination Delay 


\subsection{Lifetime of the Network}

In MANETs the lifetime of the network defines, how much time the network becomes active condition, that is how much time it perform the dedicated tasks with in a network coverage. In SCF, the Lifetime of the Network is improved. It's shown in Figure 7.

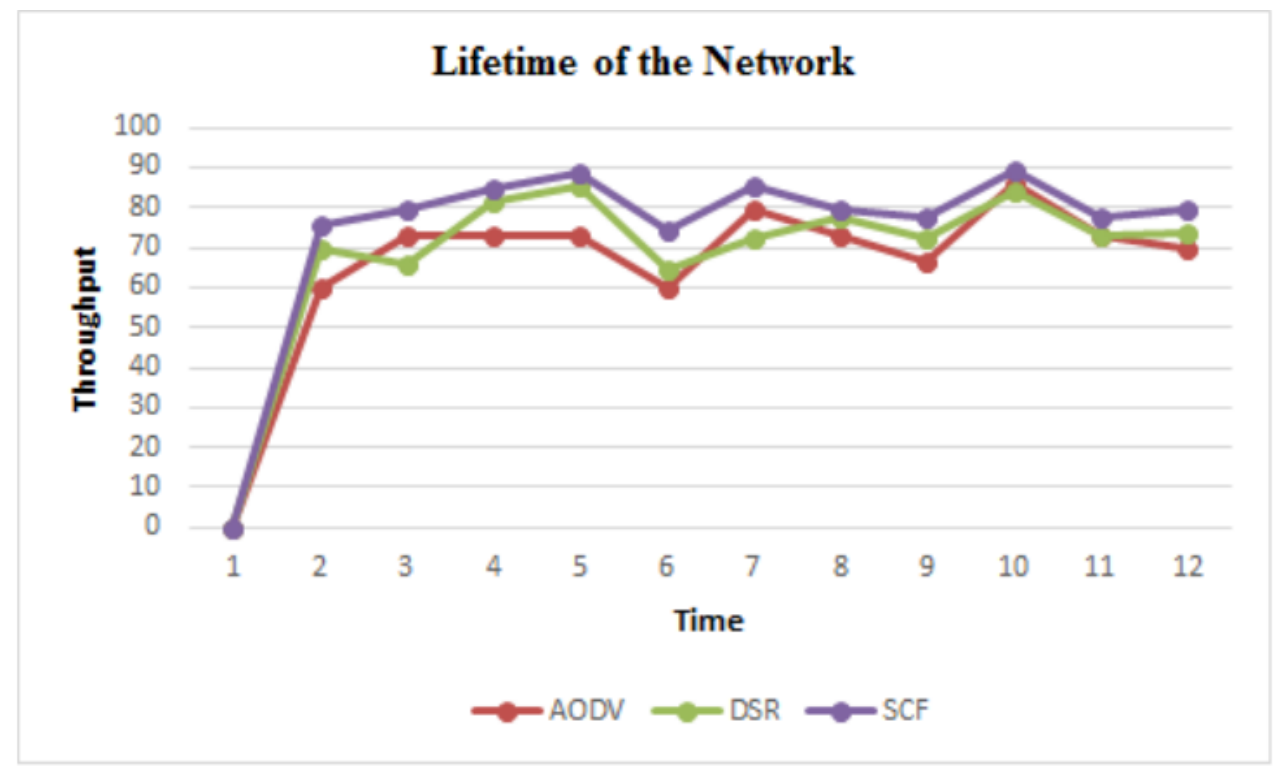

Figure 7 Lifetime of the Network

\section{CONCLUSIONS}

MANET is the emerging area of research, but still the security of MANET is not up to the level. Misbehaving nodes are compromises the networks and the malicious nodes accessed the flat architecture or infrastructure less network. In existing system the conventional replica allocation method is failed to detect the selfish nodes. It is overcome by the proposed novel replica allocation method using Self Centered Friendship tree. Through credit risk the selfish nodes are identified by SCF tree, using the unambiguous node, unambiguous query processing. The main advantage of using this proposed methodology is easy access to the end users; identify the selfish nodes in very effective manner with low operational cost. In future, to identify and intimate the communicate parties by using false alarms to the selfish nodes.

\section{REFERENCES}

[1] Ningrinla Marchang, Raja Datta and Sajal K. Das," A Novel Approach for efficient Usage of Intrusion Detection System in Mobile Ad hoc Networks", IEEE Transactions on Vehicular Technology, Vol.66,No.2,pp.1684-1695,2017

[2] E. M. Shakshuki, N. Kang and T. R. Sheltami, EAACK - a secure intrusion detection system for MANETs, IEEE Trans. Ind. Electron.2013, 1089-1098.

[3] D. Rajalakshmi, K. Meena,"A Novel based fuzzy cognitive maps protocol for intrusion discovery in Manets", International journal of recent technology and engineering, Vol.7, 2019.

[4] Srinivasan.G and Murugaanandam,'Identication of Selfishness and Efficient Replica Allocation over MANETs", International Journal of Innovations in Scientific and Engineering Research, Volume 1, Issue 2, February 2014.

[5] D.Rajalakshmi, K. Meena,"A Hybrid Intrusion Detection System for Mobile Adhoc Networks using FBID Protocol", Scalable Computing: Practice and Experience, Vol.21, No 1, 2020 . 
[6] Yuanbo Guo, Jianfeng Ma, Chao Wang and Kuiwu Yang, "Incentive-based optimal Nodes selection Mechanism for Thershold key management in MANETs with selfish nodes", International Journal of Distributed Sensor Networks Volume 2013.

[7] L. Anderegg and S. Eidenbenz,"Ad Hoc-VCG: A Truthful and Cost-Efficient Routing Protocol for Mobile Ad Hoc Networks with Selfish Agents" Proc. ACM MobiCom, pp. 245259, 2003.

[8] K. Balakrishnan, J. Deng, and P.K. Varshney, "TWOACK: Preventing Selfishness in Mobile Ad Hoc Networks", Proc. IEEE Wireless Comm. and Networking, pp. 2137-2142, 2005.

[9] D.Rajalakshmi, K. Meena,"An Efficient technique of intrusion detection for large number of malicious nodes in Manet using a tree classifier", International journal of simulation, systems, science and technology, 2018.

[10] B.-G. Chun, K. Chaudhuri, H. Wee, M. Barreno, C.H. Papadimitriou, and J. Kubiatowicz, "Selfish Caching in Distributed Systems: A Game- Theoretic Analysis". Proc. ACM Symp. Principles of Distributed Computing, pp. 21-30, 2004.

[11] L. Anderegg and S. Eidenbenz, "Ad Hoc-VCG: A Truthful and Cost-Efficient Routing Protocol for Mobile Ad Hoc Networks with Selfish Agents", Proc. ACM MobiCom, pp. 245259, 2003.

[12] G. Ding and B. Bhargava, "Peer-to-Peer File-Sharing over Mobile Ad Hoc Networks", Proc. IEEE Ann. Conf. Pervasive Computing and Comm. Workshops, pp. 104-108, 2004

[13] J.Sengathir and R.Manoharan,"Selfish Aware context based Reactive Queue Scheduling Mechanism for MANETs", International Journal of Computer Applications, Volume 66 No.19, March 2013.

[14] D. Hales, "From Selfish Nodes to Cooperative Networks - Emergent Link-Based Incentives in Peer-to-Peer Networks", Proc. IEEE Int'l Conf. Peer-to-Peer Computing, pp. 151-158, 2004.

[15] J. Broch, D.A. Maltz, D.B. Johnson, Y.-C. Hu, and J. Jetcheva, ”A Performance Comparison of Multi-Hop Wireless Ad Hoc Network Routing Protocols", Proc. ACM MobiCom, pp. 8597, 1998.

[16] Lakshmi. S, Radha. S, "Selfish aware queue scheduler for packet scheduling in MANET", In Proceedings of Recent trends in Information technology (ICRTIT), 2012.

[17] S. Anuradha, G. Raghuram, K. E. Sreenivasa murthy, B. Gurunath Reddy, "New Routing Technique to improve Transmission Speed of Data Packets in Point to Point Networks", ICGST-CNIR Journal, Volume 8, Issue 2, January 2009.

[18] D. Rajalakshmi, K. Meena, "A Survey of intrusion detection with higher malicious misbehavior detection in Manet", International journal of civil engineering and technology, Vol.8, 2017. 